Tiling a polygon with two kinds of rectangles

Olivier Bodini

Eric Rémila 


\title{
Tiling a polygon with two kinds of rectangles
}

\author{
Olivier Bodini \\ Eric Rémila
}

december 2002

\begin{abstract}
We fix two rectangles with integer dimensions. We give a quadratic time algorithm which, given a polygon $F$ as input, produces a tiling of $F$ with translated copies of our rectangles (or indicates that there is no tiling). Moreover, we prove that any pair of tilings can be linked by a sequence of local transformations of tilings, called flips.

This study is based on the use of J. H. Conway's tiling groups and extends the results of C. Kenyon and R. Kenyon (limited to the case when each rectangle has a side of length 1 ).
\end{abstract}

Keywords: tiling, tiling group, height function, flip

\section{Résumé}

Nous fixons deux rectangles aux dimensions entires. Nous produisons un algorithme en temps quadratique qui, tant donne un polygone $F$ en entre, donne un pavage de $F$ avec des copies translates de nos rectangles (ou indique qu'il n'y a pas de pavage).

De plus, nous prouvons que toute paire de pavages peur etre relie par une suite de transformations locales lmentaires.

Cette tude utilise les groupes de pavages de J. H. Conway et tend les resultats de C. Kenyon and R. Kenyon (limits au cas ou chaque rectangle a une dimension unitaire).

Mots-clés: pavage, groupe de pavage, fonction de hauteur, flip 


\title{
Tiling a polygon with two kinds of rectangles
}

\author{
Olivier Bodini * $\quad$ Eric Rémila ${ }^{\dagger}$
}

\begin{abstract}
We fix two rectangles with integer dimensions. We give a quadratic time algorithm which, given a polygon $F$ as input, produces a tiling of $F$ with translated copies of our rectangles (or indicates that there is no tiling). Moreover, we prove that any pair of tilings can be linked by a sequence of local transformations of tilings, called flips. This study is based on the use of J. H. Conway's tiling groups and extends the results of C. Kenyon and R. Kenyon (limited to the case when each rectangle has a side of length 1 ).
\end{abstract}

\section{Introduction}

In 1990, J. H. Conway and J. C. Lagarias [3] introduce the notion of tiling group. They create a way to study tiling problems with an algebraic approach. This work has been prolonged by W. P. Thurston [16], who introduces the notion of height function (also introduced independently in the statistical in the statistical physics literature (see [2] for a review)). The tools cited above have been used and generalized by different authors [4], [5], [6], [8], [11], [12], [14], [15] and some very strong algorithmic and structural results about tilings have been obtained. We have especially studied the paper from C. Kenyon and R. Kenyon [4]: in the main part, these authors give and analyze a linear algorithm which, given a polygon, produces a tiling with $m \times 1$ and $1 \times n^{\prime}$ rectangles (where $m$ and $n^{\prime}$ are some fixed integers). The end of the paper is devoted to the study of tilings with $3 \times 2$ and $2 \times 3$ rectangles. It contains the scheme of the proof (i. e. lemmas without proofs) of the correctness of a quadratic algorithm. The authors also claim (even it is no clear for the reader) that this study can be easily extended to any pair of $k \times l$ and $l \times k$ rectangles.

In the present paper, we extend the results from C. Kenyon and R. Kenyon to any pair of rectangles with integer dimensions. We give a complete proof, which avoids any ambiguity. To do it, we have to introduce a new technique (which is not necessary for $3 \times 2$ and $2 \times 3$ rectangles) to reduce the problem by cutting the polygon. Our results also prove the flip connectivity: any pair of tilings can be linked by a sequence of local transformations of tilings, called flips.

*Laboratoire de l'Informatique du Parallélisme, umr 5668 CNRS-INRIA-ENS Lyon, 46 allée d'Italie, 69364 Lyon cedex 07, France Olivier.Bodini@ens-lyon.fr

†Laboratoire de l'Informatique du Parallélisme, umr 5668 CNRS-INRIA-ENS Lyon, 46 allée d'Italie, 69364 Lyon cedex 07, France and GRIMA, IUT Roanne, Université Jean Monnet Saint-Etienne 20 avenue de Paris, 42334 Roanne cedex, France Eric.Remila@ens-lyon.fr 
The paper is divided as follows: we first give the definitions and basic concepts (section 2). Afterwards, we explain our new techniques to cut tilings (section 3) and we show how to combine them with the machinery of height functions on tilings (section 4) to get a quadratic tiling algorithm and prove the flip connectivity. We finish with the particular cases where at least a side of a rectangle is 1 (some simplifications appear, section 5) and with some extensions and open problems (section 6).

\section{Basic tools}

\subsection{The square lattice}

let $\Lambda$ be the square lattice of the Euclidean plane $\mathbb{R}^{2}$. A cell of $\Lambda$ is a square of unites sides whose vertices haves integer coordinates. The vertices and edges of $\Lambda$ are those of the cells of $\Lambda$. Two vertices are neighbors if they are linked by an edge.

A path $P$ of $\Lambda$ is a finite sequence of vertices such that two consecutive vertices are neighbors. A corner of a path $P$ is the origin or final vertex, or a vertex $v$ of $P$ which is not equal to the center of the line segment linking the predecessor and the successor of $v$ in $P$. A corner $v$ is weak if its predecessor and its successor are equal, otherwise the corner is strong (the origin and final vertices of the path are strong corners). A path can be defined by the sequence $\left(v_{0}, v_{1}, \ldots, v_{p}\right)$ of its corners. In this paper, we encode paths by this way. A move of a path is the subpath linking two consecutive corners. We have two kinds of moves: the horizontal and vertical ones. The algebraic length of a horizontal (respectively vertical) move from $v_{i}=\left(x_{i}, y_{i}\right)$ to $v_{i+1}=\left(x_{i+1}, y_{i+1}\right)$ is the difference $x_{i+1}-x_{i}$ (respectively $y_{i+1}-y_{i}$ ).

A (finite) figure $F$ of $\Lambda$ is a (finite) union of (closed) cells of $\Lambda$. Its boundary is denoted by $\delta F$. The lower boundary is the union of horizontal line segments $] v, v^{\prime}[$ included in $\delta F$, such that there exists a rectangle whose bottom side is $\left[v, v^{\prime}\right]$, included in $F$. On defines in a similar way the left, right, and upper boundaries of $F$. A figure $F$ is simply connected if $F$ and its complement $\mathbb{R}^{2} \backslash F$ both are connected. A finite simply connected figure $F$ is called a polygon of $\Lambda$. The boundary of a polygon $F$ canonically induces a cycle in $\Lambda$, which is called the boundary cycle of $F$. In this paper (even when this is not explicitly written), we only consider polygons.

\section{$2.2 \quad$ Tilings}

We fix a pair of $\left\{R, R^{\prime}\right\}$ of rectangles with vertical and horizontal sides and integer dimensions. The width (i. e. horizontal dimension) of $R$ (respectively $R^{\prime}$ ) is denoted by $m$ (respectively $m^{\prime}$ ), the height (i. e. vertical dimension) of $R$ (respectively $R^{\prime}$ ) is denoted by $n$ (respectively $n^{\prime}$ ). An $R$-tile (respectively $R^{\prime}$-tile) is a translated copy of the rectangle $R$ (respectively the rectangle $R^{\prime}$ ). A tiling $T$ of a figure $F$ is a set of tiles included in $F$, with pairwise disjoint interiors (i. e. there is no overlap), such that the union of the tiles of $T$ equals $F$ (i. e. there is no gap). A tile $t$ is cut by a path $P$ if there exists an open line segment $] v, v^{\prime}$ [ linking two consecutive vertices of $P$, included in the interior part of the tile $t$. The subgraph $G_{T}=\left(V_{T}, E_{T}\right)$ of $\Lambda$ such that $V_{T}$ (respectively $E_{T}$ ) is the set (respectively edges) of vertices of $\Lambda$ which are on the boundary of a tile of 
$T$. Obviously, the set $V_{T}$ (respectively $E_{T}$ ) contains all the vertices (respectively edges) of $\Lambda$ which are in $\delta F$.

\subsection{The preprocessing}

The problem of tiling can easily be reduced to the case when $\operatorname{gcd}\left(m, m^{\prime}\right)=\operatorname{gcd}\left(n, n^{\prime}\right)=$ 1 as follows : consider the only tiling $\Theta$ of the figure $F$ with translated copies of a $\operatorname{gcd}\left(m, m^{\prime}\right) \times \operatorname{gcd}\left(n, n^{\prime}\right)$ rectangles, called bricks . The tiling $\Theta$ is a raffinement of any "classical" tiling $T$ with $R$-tiles and $R^{\prime}$-tiles, i. e. each brick of $\Theta$ is included in a tile of $T$.

Two bricks $\theta$ and $\theta^{\prime}$ of $\Theta$ are called equivalent if they respectively are the first and last element of a finite sequence of bricks such that two consecutive bricks of the sequence share a whole side. Remark that two bricks included in a same tile of $T$ are equivalent (i. e. the boundary of an equivalence class cuts no tile of $T$ ), and the union of all the bricks of an equivalence class form a polygon (if such a union had a hole, then a brick placed on a corner of the hole would create a contradiction). Thus, the problem can be simplified as follows: We first compute the equivalence classes(this can easily be done in a linear time in the area of the polygon). Afterwards, we have to study tilings on each equivalence class. But, since in each class, bricks are equivalent, we can change the units in order to consider a brick as the new unit rectangle.

This transformation makes us consider that $\operatorname{gcd}\left(m, m^{\prime}\right)=\operatorname{gcd}\left(n, n^{\prime}\right)=1$. In the rest of the paper, we will assume that this simplification has been done and thus $\operatorname{gcd}\left(m, m^{\prime}\right)=$ $\operatorname{gcd}\left(n, n^{\prime}\right)=1$.

\section{Tiling groups and cut lines}

The two following sections are devoted to the main case, called the non-degenerate case, when we have $m>1, n>1, m^{\prime}>1$ and $n^{\prime}>1$.

We introduce the groups $G_{1}=\mathbb{Z} / m \mathbb{Z} * \mathbb{Z} / n^{\prime} \mathbb{Z}$ (i. e. the free product (see for example [7] for details about group theory) of both groups $\mathbb{Z} / m \mathbb{Z}$ and $\mathbb{Z} / n^{\prime} \mathbb{Z}$ ), and $G_{2}=\mathbb{Z} / m^{\prime} \mathbb{Z} *$ $\mathbb{Z} / n \mathbb{Z}$. We only give properties and notations for $G_{1}$, since they are symmetric for $G_{2}$. We recall that there exists a canonical surjection (denoted by $s_{1}$ ) from $G_{1}$ to the Cartesian product $\mathbb{Z} / m \mathbb{Z} \times \mathbb{Z} / n^{\prime} \mathbb{Z}$, and that each element $g$ of $G_{1}$ can be written in a unique way: $g=\prod_{i=1}^{p} g_{i}$, with, for each integer $i$ such that $1 \leq i \leq p, g_{i} \neq 1_{G_{1}}$ and for each integer $i$ such that $1 \leq i<p, g_{i}$ is element of $\mathbb{Z} / m \mathbb{Z}$ if and only if $g_{i+1}$ is element of $\mathbb{Z} / n^{\prime} \mathbb{Z}$. This expression is called the canonical expression of $g$ and the integer $p$ is called the length of $g$ (denoted by $l(g)$ ). By this way, an order relation is created on $G_{1}$ : we say that $g \leq g^{\prime}$ if the canonical expression of $g$ is a prefix of $g^{\prime}$.

Two distinct elements $g$ and $g^{\prime}$ of $G_{1}$ are $H$-equivalent if there exists an element $x$ of $\mathbb{Z} / m \mathbb{Z}$ such that $g^{\prime}=g x$, they are $V$-equivalent if there exists an element $y$ of $\mathbb{Z} / n^{\prime} \mathbb{Z}$ such that $g^{\prime}=g y$, they are neighbors if they are $H$-equivalent or $V$-equivalent. Each element of $G_{1}$ is exactly the intersection of an $H$-equivalence class and a $V$-equivalence class.

The graph of $G_{1}$ is the symmetric graph whose vertices are elements of $G_{1}$, and for each pair $\left(g, g^{\prime}\right)$ of distinct elements of $G_{1},\left(g, g^{\prime}\right)$ is an edge if and only if $g$ and $g^{\prime}$ are 
neighbors. ${ }^{1}$ Remark that each path of this graph linking an element $g$ to an element $g^{\prime}$ has to pass through each element $g^{\prime \prime}$ such that $1_{G_{1}} \leq g^{-1} g^{\prime \prime} \leq g^{-1} g^{\prime}$. Informally, we can say that the structure of graphs elements is an infinite tree of equivalent classes.

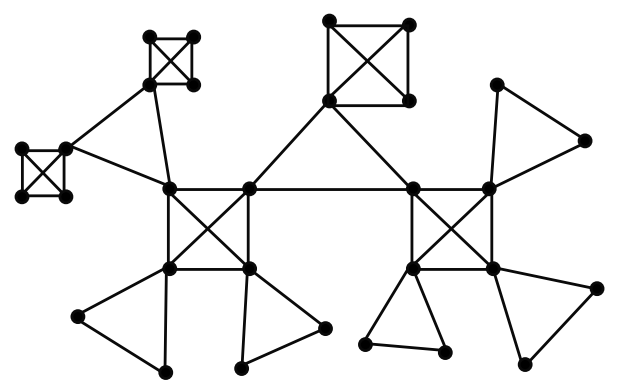

Figure 1: The graph of $G_{1}$.

Let $P=\left(v_{1}, \ldots, v_{p}\right)$ (according to our convention, the vertices of the sequence are the corners of $P$ ). This path canonically induces an element $g_{P}$ of $G_{1}$ as follows: each horizontal move gives an element of $\mathbb{R} / m \mathbb{Z}$ (equal to its algebraic length), each vertical move gives an element of $\mathbb{R} / n^{\prime} \mathbb{Z}$ and the element $g_{P}$ is obtained successively making the product of elements given by successive moves. Notice that any path describing the contour of a tile induces the unit element $1_{G_{1}}$. Thus, tilings can be encoded using the groups $G_{1}$ and $G_{2}$, as we see below.

Definition 1 (tiling function) Let $T$ be a tiling of a polygon $F$. A tiling function induced by $T$ is a mapping $f 1_{T}$ from the set $V_{T}$ to $G_{1}$ such that, for each edge $[v=$ $\left.(x, y), v^{\prime}=\left(x^{\prime}, y^{\prime}\right)\right]$ cutting no tile of $T$ :

* if $x=x^{\prime}$ then $f 1_{T}\left(v^{\prime}\right)=f 1_{T}(v)\left(y^{\prime}-y\right)$, with $y^{\prime}-y$ taken as an element of $\mathbb{Z} / n^{\prime} \mathbb{Z}$,

* if $y=y^{\prime}$ then $f 1_{T}\left(v^{\prime}\right)=f 1_{T}(v)\left(x^{\prime}-x\right)$, with $x^{\prime}-x$ taken as an element of $\mathbb{Z} / m \mathbb{Z}$,

Proposition 1 (J. H. Conway) let $F$ be a polygon, $T$ be a tiling of $F$, $v_{0}$ be a vertex of the boundary of $F$ and $g_{0}$ be a vertex of $G_{1}$. If $F$ is connected (respectively is a polygon), then there exists at most one (respectively exactly one) tiling function $f 1_{T}$ induced by $T$ such that $f 1_{T}\left(v_{0}\right)=g_{0}$.

Proof. (sketch) The function $f 1_{T}$ can easily be constructed successively exploring the contour of each tile: the function $f 1_{T}$ is first defined on the vertices of a tile $t_{0}$ which has $v_{0}$ on its boundary. Afterwards, $f 1_{T}$ is defined on the vertices of a tile $t_{1}$ which shares a part of its boundary with $t_{0}$ and so on. This method gives the uniqueness of $f 1_{T}$, for $F$ connected (nevertheless, a conflict (i. e. a vertex $v$ such that two distinct values of $f 1_{T}(v)$ are given) can arise if $F$ has some holes, which yields that there is no tiling function).

The existence for polygons is proved by induction on the number of tiles of $T$. If $F$ is a polygon, then either $F$ is reduced to a tile (and the existence is trivial) or $F$ can be cut into two sub-polygons $F_{1}$ and $F_{2}$, in such a way that each tile of $T$ is included in either $F_{1}$ or $F_{2}$. One can assume without loss of generality that $v_{0}$ is in $F_{1}$, thus, by induction hypothesis, $f 1_{\left.T\right|_{F_{1}}}$ exists on $F_{1}$. Now, take a point $v_{1}$ at the boundary of both

\footnotetext{
${ }^{1}$ The graph of elements is the Cayley graph of $G_{1}$ when the set of generators is $(\mathbb{Z} / m \mathbb{Z})^{*} \cup\left(\mathbb{Z} / n^{\prime} \mathbb{Z}\right)^{*}$ and labels have been removed.
} 
$F_{1}$ and $F_{2}$. By induction hypothesis, there also exists a tiling function $f 1_{T_{F_{2}}}$ on $F_{2}$ such that $f 1_{\left.T\right|_{F_{1}}}\left(v_{1}\right)=f 1_{\left.T\right|_{F_{2}}}\left(v_{1}\right)$. This gives the existence of the tiling function in the whole polygon $F$.

We will now study tilings of a polygon $F$. For each tiling $T$, we use the unique tiling function $f 1_{T}$ such that $f 1_{T}(O)$ is the unit element of $G_{1}$, where $O$ denotes a fixed lower left corner of the boundary of $F$. Remark that, for each vertex $v$ on $\delta F, f 1_{T}(v)$ does not depend on the tiling $T$ (which allows to remove the tiling index and write $f 1(v)$ ) and can be computed without the knowledge of $T$, following the boundary of $F$. We also note that, for each point $v$ of $V_{T}$, the value $s_{1} \circ f 1_{T}(v)$ is the pair of coordinates of $v$ (respectively modulo $m$ and modulo $n^{\prime}$ ), and, consequently, does not depend on $T$.

\subsection{Cutting using algebraic constraints}

Definition $2 A$ special path $P$ of $F$ is a a path defined by a sequence $\left(v_{1}, \ldots, v_{p}\right)$ of successive corners such that:

* the vertex $v_{1}$ is on the lower boundary of $F$, the vertex $v_{p}$ is on the boundary of $F$, and for each integer $i$ such that $1 \leq i<p$, the line segment $\left[v_{i}, v_{i+1}\right]$ is included in $F$,

* for each odd integer $i$ such that $1 \leq i<p$, there exists a non-null integer $k_{i}$ such that $-m^{\prime}<k_{i}<m^{\prime}$ and $v_{i+1}=v_{i}+\left(k_{i} m, 0\right)$,

* for each even integer $i$ such that $1<i<p$, there exists a non-null integer $k_{i}$ such that $-n<k_{i}<n$ such that $v_{i+1}=v_{i}+\left(0, k_{i} n^{\prime}\right)$,

* the element $\left(f 2\left(v_{0}\right)\right)^{-1} f 2\left(v_{p}\right)$ is the element $g_{P}$ of $G_{2}$ induced by the path $P, i$. e. $\Pi_{i=1}^{p-1} r_{i} k_{i}$, with $r_{i}=n^{\prime}$ for $i$ even and $r_{i}=m$ for $i$ odd.

The notion of special path with the proposition below form the main new idea of this paper. There exists a quite easier proof in the particular case when $m^{\prime}=n=2$ (sketched in [4]) since the graph of elements has a line structure.

Proposition 2 Let $T$ be a tiling and $P$ be a special path of a polygon $F$. The path $P$ cuts no tile of $T$.

Proof. We first construct from $P$ another path $P_{T}$ as follows: for each tile $T$ which is cut by $P$, let $P_{t, \text { cross }}$ denote the sub-path of $T$ which crosses $t$. The path $P_{T}$ is obtained replacing in $P$ each sub-path $P_{t, \text { cross }}$ by the shortest path $P_{t, \text { surround }}$, on the boundary of $t$, with the same endpoints as $P_{t, c r o s s}$ (if two paths are possible, any convention is possible; one can state, for example, that $t$ is surrounded by left). Notice that $P_{t, \text { surround }}$ is formed from two or three moves. Informally, The path $P_{T}$ is an approximation of $P$ which cuts no tile of $T$.

Let $i$ be an integer such that $1 \leq i \leq p$. We define the vertex $v_{i}^{\prime}$ such that, $v_{i}^{\prime}$ is the last strong corner of $P_{T}$ such that $f 2_{T}\left(v_{i}^{\prime}\right)$ is equal to the element $g_{p_{i}}$ induced by the sub-path of $P$ from $v_{0}$ to $v_{i}$ (i. e. $g_{p_{i}}=\prod_{j=0}^{i} r_{j} n_{j}$ (with $r_{j}=n^{\prime}$ for $i$ even and $r_{j}=m$ for $j$ odd)). This corner exists because of the structural properties of the graphs of elements and classes of $G_{2}$ (especially the tree structure of the graph of classes). The point $v_{i}^{\prime \prime}$ is 
defined as the last point reached in $P_{T}$, before $v_{i}^{\prime}$ which also is in $P$ (possibly $v_{i}^{\prime \prime}=v_{i}^{\prime}$; see figure 2 for the notations). We claim the following fact:

Fact: the vertex $v_{i}$ is before $v_{i}^{\prime \prime}$ in $P$ and, if, moreover, $v_{i}=v_{i}^{\prime}$ then no tile of $T$ is cut by $P$ before this vertex.

The above fact is proved by induction. The result is obviously true for $i=1$. Now, assume that it also holds for a fixed integer $i$ such that $1 \leq i<p$.

If $v_{i+1}$ is before $v_{i}^{\prime \prime}$ on $P$, the result is also obvious, since $v_{i}^{\prime \prime}$ is before $v_{i+1}^{\prime \prime}$. Moreover, remark that, for each vertex $v, s_{2} \circ f 2_{T}\left(v_{i}^{\prime}\right)$ is equal to the pair of coordinates of $v$ (respectively modulo $m^{\prime}$ and $n$ ), thus $s_{2} \circ f 2_{T}\left(v_{i}\right) \neq s_{2} \circ f 2_{T}\left(v_{i+1}\right)$, because of the first component. On the other hand we have $s_{2} \circ f 2_{T}\left(v_{i}^{\prime}\right)=s_{2} \circ f 2_{T}\left(v_{i}\right)$. Thus $v_{i}^{\prime} \neq v_{i+1}$, which yields that $\left.v_{i+1} \neq v_{i+1}^{\prime}\right)$.

Otherwise, by induction hypothesis, the point $v_{i}^{\prime \prime}$ is on the line segment $\left[v_{i}, v_{i+1}\right]$. We only treat the case when $i$ is odd and $k_{i}$ is positive, since the proof is similar in the other cases.

Since $v_{i}^{\prime}$ is a strong corner of $P_{T}, v_{i}^{\prime}$ is a left side of a tile $t_{1}$ used to define $P_{T}$. The vertex $v_{i}^{\prime \prime}$ is also on the left side of $t_{1}$, thus they have have the same horizontal coordinate. Assume that $v_{i+1}^{\prime \prime}$ is before $v_{i+1}$ in $P$. In this case, $v_{i+1}^{\prime}$ is a right vertex of a tile of $T$, and the vertical line passing through $v_{i+1}^{\prime}$ cuts the line segment $\left[v_{i}, v_{i+1}\right]$ in a vertex $v_{i+1}^{\prime \prime \prime}$.

Now consider the line segment $\left[v_{i}^{\prime \prime}, v_{i+1}^{\prime \prime \prime}\right]$. This segment is not reduced to a vertex (since $v_{i}^{\prime}$ and $v_{i+1}^{\prime}$ have not the same horizontal component modulo $m^{\prime}$ ). Moreover, there exists a sequence $\left(t_{1}, t_{2}, \ldots, t_{q}\right)$ of tiles of $T$ such that the left side of $t_{1}$ contains $v_{i}^{\prime}$ and $v_{i}^{\prime \prime}$, the right side of $t_{q}$ contains $v_{i+1}^{\prime}$ and $v_{i+1}^{\prime \prime \prime}$, and for each integer $j$ such that $0 \leq j<q$, the intersection of the right side of $t_{i}$ and the left side of $t_{i+1}$ is a line segment non reduced to a single point. Let $\mu$ (respectively $\mu^{\prime}$ ) denote the number of $R$-tiles (respectively $R^{\prime}$-tiles) on the sequence $\left(t_{1}, t_{2}, \ldots, t_{q}\right)$. We have, using the horizontal coordinates:

$$
\mu m+\mu^{\prime} m^{\prime} \leq k_{i} m
$$

and, from the definition of $v_{i}^{\prime \prime}$ and $v_{i+1}^{\prime \prime \prime}$ :

$$
\mu m+\mu^{\prime} m^{\prime}=k_{i} m\left[m^{\prime}\right]
$$

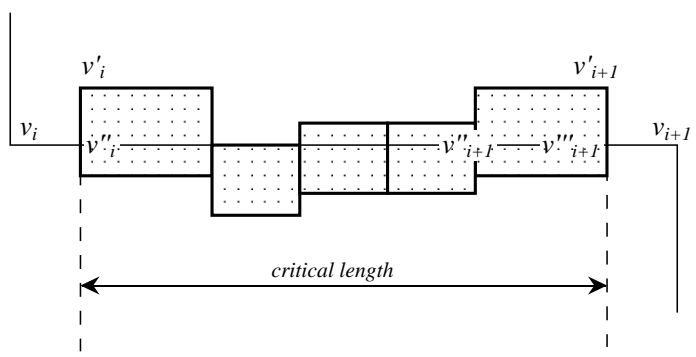

Figure 2: The main argument used in the proof of Proposition 2. The critical length has to be equal (modulo $m^{\prime}$ ) to the length of the line segment $\left[v_{i}, v_{i+1}\right]$ and, on the other hand, this length is the sum of widths of tiles.

Thus, we necessarily have $\left(\mu, \mu^{\prime}\right)=\left(k_{i}, 0\right)$ (since $\operatorname{gcd}(m, n)=1$, the equality 2 gives that $\mu=k_{i}+k m^{\prime}$ with $k \in \mathbb{Z}$, the integer $k$ is null since $0<\mu<m^{\prime}$ and $\mu^{\prime}=0$ from the equality 1) 
This means that $v_{i}^{\prime \prime}=v_{i}, v_{i+1}^{\prime \prime \prime}=v_{i+1}$ and the line segment $\left[v_{i}, v_{i+1}\right]$ only cuts $R$-tiles.

Thus $v_{i}^{\prime}$ and $v_{i}$ both are on the left side of the $R$-tile $t_{1}$ which yields that $v_{i}^{\prime}=v_{i}$ since their vertical coordinates are equal modulo $n$. With the same argument, we necessarily have $v_{i+1}^{\prime}=v_{i+1}$. Thus we have

$$
f 2_{T}\left(v_{i+1}\right)=f 2_{T}\left(v_{i}\right)\left(k_{i} m\right)
$$

Now, consider the path starting in $v_{i}$ by a horizontal move which follows the boundary of the polygon formed by the $k_{i} R$-tiles of the sequence, until $v_{i+1}$ in reached. This path directly induces a word, without possible simplification in $G_{2}$ (since the (absolute) length of each vertical move is lower than $n$, and the length of each horizontal move is $\mathrm{km}$ with $k$ integer such that $0<k \leq k_{i}<m^{\prime}$ ). This word is necessarily $k_{i} m$, from the equality 3. This means that no tile is cut by the segment $\left[v_{i}, v_{i+1}\right]$. This finishes the proof of the fact above, using induction.

Now, to get the result, it suffices to apply the fact for $i=p$. We necessarily have: $v_{p}^{\prime}=v_{p}$ since $v_{p}$ finishes the path, thus the whole path cuts no tile.

\subsection{Application to stairs of blocks}

Definition $3 A$ horizontal block (respectively vertical block) of $F$ is a (closed) $m m^{\prime} \times n^{\prime}$ rectangle (respectively $m \times n n^{\prime}$ rectangle).

$A$ horizontal (respectively vertical) block $B$ is covered by a block $B^{\prime}$ if the (open) top (respectively right) side of $B$ and the (open) bottom (respectively left) side of $B$ share a (non-reduced to a vertex) line segment of length $\mathrm{km}$, where $k$ denotes a positive integer.

$A$ stair of blocks (see figure 3) is a finite sequence $\left(B_{1}, B_{2}, \ldots, B_{p}\right)$ of blocks of the same type such that, for each integer $i$ such that $1 \leq i<p, B_{i+1}$ covers $B_{i}$, and there exists no subsequence $\left(B_{i+1}, B_{i+2}, \ldots, B_{i+k}\right)$ such that $\cup_{j=1}^{k} B_{i+j}$ is an $\mathrm{mm}^{\prime} \times n n^{\prime}$ rectangle.

Notice that the figure formed by the union of blocks of a fixed stair has exactly one tiling which contains a unique type of tiles.

Proposition 3 Assume that there exists a stair $\left(B_{1}, B_{2}, \ldots, B_{p}\right)$ of vertical blocks such that the intersection of $B_{1}$ with the lower boundary of $F$ contains a horizontal line segment $S$ of length at least $m$, the intersection of $B_{p}$ with the upper boundary of $F$ contains a horizontal line segment $S^{\prime}$ of length at least $m$, and the tiles of the tiling of $\cup_{i=1}^{p} B_{i}$ can be placed in $F$ without inducing contradiction in the definition of the tiling function.

Each tiling $T$ of $F$ contains the tiling of $\cup_{i=1}^{p} B_{i}$.

Proof. From the hypothesis, there exists a a vertex $v$ of $S$ such that the distance between $v$ and the lower right corner of $B_{1}$ is $k m$, for an integer $k$ such that $0<k<m^{\prime}$. On a similar way, there exists a vertex $v^{\prime}$ of $S^{\prime}$ such that the distance between $v^{\prime}$ and the upper right corner of $B_{p}$ is $k^{\prime} m$, for an integer $k^{\prime}$ such that $0<k^{\prime}<m^{\prime}$.

The path from $v$ to $v^{\prime}$ following the boundary of $\cup_{i=1}^{p} B_{i}$ counterclockwise is a special path and, from the previous proposition, no tile of $T$ cuts this path. The same argument can be used following the boundary of $\cup_{i=1}^{p} B_{i}$ clockwise. This gives that no tile of $T$ is cut by the boundary of $\cup_{i=1}^{p} B_{i}$.

Of course, we have a similar proposition for stairs of vertical blocks. 


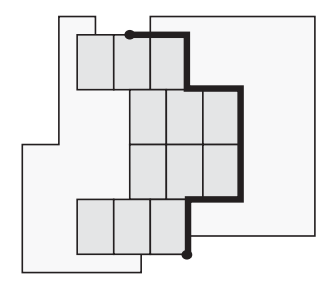

Figure 3: A stair of blocks and an associated special path

\section{Height function and order}

Definition 4 (height, predecessor, order on $\left.G_{1}\right)$ The height $h 1(g)$ of an element $g$ of $G_{1}=\mathbb{Z} / m \mathbb{Z} * \mathbb{Z} / n^{\prime} \mathbb{Z}$ is defined by the following axioms :

* if there exists an integer $k$ such that the canonical expression of $g$ is $\left(m^{\prime} n\right)^{k}$ or $\left(m^{\prime} n\right)^{k} m^{\prime}$, then $h 1(g)=-l(g)$ (with $m^{\prime}$ taken as an element of $\mathbb{Z} / m \mathbb{Z}$ and $n$ taken as an element of $\left.\mathbb{Z} / n^{\prime} \mathbb{Z}\right)$,

* for each element $g$, there exists a unique element $\operatorname{pr}(g)$ (called the predecessor of $g$ ) equivalent to $g$, such that $h 1(\operatorname{pr}(g))=h 1(g)-1$,

* for each element $g^{\prime}$, equivalent to both $g$ and $\operatorname{pr}(g)$, we have : $h 1\left(g^{\prime}\right)=h 1(g)$.

* for each other neighbor $g^{\prime \prime}$ of $g$, we have $h 1\left(g^{\prime \prime}\right)=h 1(g)+1$.

Given a pair $\left(g, g^{\prime}\right)$ of elements of $G_{1}$, we say that $g \leq g^{\prime}$ if there exists a sequence $\left(g_{1}, g_{2}, \ldots, g_{p}\right)$ of elements of $G_{1}$ such that $g_{1}=g, g_{p}=g^{\prime}$ and, for for each integer $i$ such that $0 \leq i<p, g_{i}$ is the predecessor of $g_{i+1}$.

Notice that, with the notations above, we have $g=\operatorname{pr}\left(g^{\prime \prime}\right)$. Also notice that the canonical expression of any element $g$ can be written $w_{1} w_{2}$, where $w_{1}$ is a prefix word of maximal length such that $w_{1}=\left(m^{\prime} n\right)^{k}$ or $w_{1}=\left(m^{\prime} n\right)^{k} m^{\prime}$, for an integer $k$. From this decomposition, $h 1(g)$ can be computed easily:

$* h 1(g)=l(w)$ if $w_{1}$ is the empty word,

$* h 1(g)=-l(w)$ if $w_{2}$ is the empty word,

$* h 1(g)=l\left(w_{2}\right)-l\left(w_{1}\right)-1$ otherwise.

Notice that the heights of vertices which are on the same side $\left[v^{\prime}, v^{\prime \prime}\right]$ of a tile form either a singleton $\{h\}$ or a pair $\{h, h-1\}$. With these notations, we say that $h$ is a the height of $\left[v^{\prime}, v^{\prime \prime}\right]$ in $T$ (denoted by $h 1_{T}\left(\left[v^{\prime}, v^{\prime \prime}\right]\right)$. With the convention : $f 1_{T}(O)=1_{G_{1}}$, the height of horizontal sides is even, and the height of vertical sides is odd. Both vertical sides of an $R$-tile $t$ have the same height, say $h$ and the side of each horizontal tile is either $h-1$ or $h+1$ (a symmetric situation holds for $V$-tiles). The height $h 1(t)$ of the tile $t$ is defined by : $h 1(T)=h$ (i. e. the height of an $R$-tile (respectively $R^{\prime}$-tile) is the height of its vertical (respectively horizontal) sides. The height $h 1(T)$ of the tiling $T$ is defined by: $h 1(T)=\sum_{t \in T} a(t) h 1_{T}(t)$ where $a(t)$ denotes the area of the tile $t$.

\subsection{Local flips}

Weak flips: An $m m^{\prime} \times\left(n+n^{\prime}\right)$ rectangle admits two tilings. Let $T$ be a tiling of $F$ such that an $m m^{\prime} \times\left(n+n^{\prime}\right)$ rectangle is exactly covered by tiles of $T$. The replacement 
of those tiles of $T$ (which cover the $m m^{\prime} \times\left(n+n^{\prime}\right)$ rectangle) by tiles of the other tiling of the same rectangle is called a weak flip (see figure 4 ). By this way, a new tiling $T_{\text {flip }}$ is obtained.

The flip is said pushing up if $R^{\prime}$-tiles of $T$ are in the bottom part of the $m m^{\prime} \times\left(n+n^{\prime}\right)$ rectangle (and $R^{\prime}$-tiles of $T_{\text {flip }}$ are in the top part of the $m m^{\prime} \times\left(n+n^{\prime}\right)$ rectangle). Otherwise, the flip is said pushing down.

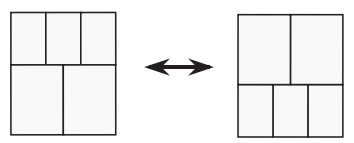

Figure 4: A weak flip

If the height of both horizontal sides of the $m m^{\prime} \times\left(n+n^{\prime}\right)$ rectangle differ by two units, then $h 1(T)$ and $h 1\left(T_{\text {flip }}\right)$ are not equal. Otherwise $h 1(T)=h 1\left(T_{\text {flip }}\right)$ and the flip is said height stable.

In a symmetric way, we have another type of weak flips, based on the two tilings of an $\left.\left(m+m^{\prime}\right) \times n n^{\prime}\right)$ rectangles. We use some similar definitions (pushing left flip, pushing right flip, height stable flip).

Strong flips: We have another type of flip (called strong flip) based on the fact that we have two ways to tile a $m m^{\prime} \times n n^{\prime}$ rectangle (see figure 5). Such a flip always changes the value of $h 1(T)$.

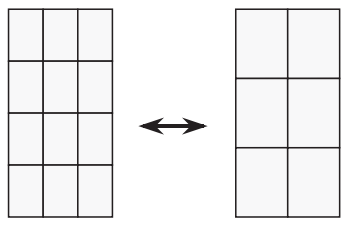

Figure 5: A strong flip

Flips permit to introduce an equivalence and an order relation on tilings as follows:

\section{Definition 5 (equivalence, order on the set of tilings)}

Two tilings are said height equivalent if one can pass from one to the other one by a sequence of height stable flips.

Given a pair $\left(T, T^{\prime}\right)$ of tilings, we say that $T$ covers $T^{\prime}$ is there exists a pair $\left(T_{\text {aux }}, T_{\text {aux }}^{\prime}\right)$ of tilings of $F$ such that $T$ is equivalent to $T_{\text {aux }}, T^{\prime}$ is equivalent to $T_{\text {aux }}^{\prime}, h 1\left(T_{\text {aux }}\right)>$ $h 1\left(T_{a u x}^{\prime}\right)$, and $T_{a u x}$ is deduced from $T_{\text {aux }}^{\prime}$ by one flip. The order relation which is the transitive closure of the relation of covering is denoted by $>$.

\subsection{Critical tiling}

For the order defined above. There exists at least a minimal tiling (since the set of tilings is finite) and if a tiling is minimal, then all the height equivalent tilings are also minimal.

Thus there exists a critical tiling $T_{\text {crit }}$, i. e. a minimal tiling such that neither height equivalent pushing down flip nor height equivalent pushing left flip can be done. To prove that such a tiling exists, it suffices to consider consider the potential function pot defined 
as follows: for each tiling $T, \operatorname{pot}(T)$ is the sum of the vertical coordinates of the horizontal sides of $R^{\prime}$-tiles of $T$ and the horizontal coordinates of the vertical sides of $R^{\prime}$-tiles. Notice that a pushing down (respectively left) flip removes $2 m n^{\prime}$ (respectively $2 m^{\prime} n$ ) units to the function pot. Thus, for any weak flip $\operatorname{pot}(T)$ and $\operatorname{pot}\left(T_{\text {flip }}\right)$ are comparable. A minimal tiling which has the lower potential value among the minimal tiling is necessarily critical.

Proposition 4 Let $T_{\text {crit }}$ be a critical tiling. Let $M$ (respectively $M^{\prime}$ ) denote the highest value of $h 1_{T_{\text {crit }}}$ in $V_{T_{\text {crit }}}$ (respectively $\left.\delta F\right)$. We have: $M^{\prime} \geq M-1$. Moreover, when $M^{\prime}=M-1$ and $M$ is odd:

* there exists a set of blocks with pairwise disjoined interiors (called critical blocks) whose tilings are included in $T_{\text {crit }}$, for each vertex $v, h 1_{T_{\text {crit }}}(v)=M$ if and only if $v$ is in the interior part of a line segment $\left[v^{\prime}, v^{\prime \prime}\right]$ which is the common side of two $R^{\prime}$-tiles of the same block.

* each critical block of $T_{\text {crit }}$ whose bottom side does not share a line segment with $\delta F$ covers another critical block of $T_{\text {crit }}$

* there is no set of $n$ critical blocks whose union is an $m m^{\prime} \times n n^{\prime}$ rectangle,

* the value $M^{\prime}$ is reached on both lower and upper boundaries of $F$.

Proof. Assume that $M>M^{\prime}$ and $M$ is odd. Let $v$ be a vertex such that $h 1_{T}(v)=M$. The point $v$ cannot be the corner of a tile of $T$, and for each side $\left[v^{\prime}, v^{\prime \prime}\right]$ of a tile $t$ of $T$ containing $v$, we necessarily have $f 1_{T}\left(v^{\prime}\right)=f 1_{T}\left(v^{\prime \prime}\right)=\operatorname{pr}\left(f 1_{T}(v)\right)$. On the other hand, this side is vertical, since $M$ is odd. This yields that $t$ is an $R^{\prime}$-tile. Moreover, since $v$ is an interior point of $F$, then $\left[v^{\prime}, v^{\prime \prime}\right]$ is shared by two $R^{\prime}$-tiles, whose height of both horizontal sides of a is $M-1$.

Assume that $t$ is the $R^{\prime}$-tile whose left side is $\left[v^{\prime}, v^{\prime \prime}\right]$. Let $k$ denote the lowest integer such that $f 1_{T}\left(v^{\prime}\right)\left(k m^{\prime}\right)=\operatorname{pr}\left(f 1_{T}(v)\right.$ ) (with $m^{\prime}$ seen as an element of $\mathbb{Z} / m \mathbb{Z}$ ). The vertex $v^{\prime}+\left(m^{\prime}, 0\right)$ is a right corner of $t$ and $f 1_{T}\left(v^{\prime}+\left(m^{\prime}, 0\right)\right)=f 1_{T}\left(v^{\prime}\right) m^{\prime}$ (with $m^{\prime}$ seen as an element of $\mathbb{Z} / m \mathbb{Z}$ ). Thus, if $k>1$, then the height of the vertices on the (open) right side of $t$ is $M$, thus $t$ shares its right side with another $R^{\prime}$-tile $t_{1}$. If $k>2$, then the argument can be repeated. The same type argument can also be used on the left side of $t$. By this way, we have a set $\left\{t_{k-m}, t_{k-m+1}, \ldots, t_{-1}, t, t_{1}, \ldots, t_{k-1}\right\}$ of $R^{\prime}$-tiles of $T_{\text {crit }}$ whose union form a critical block. This proves the first item of the proposition.

We now take a critical block of $T_{\text {crit }}$ and we are interested in what happens below its bottom side $\left[u^{\prime}, u^{\prime \prime}\right]$. Assume that the boundary $\delta F$ is not reached in (at least) a subsegment of $\left[u^{\prime}, u^{\prime \prime}\right]$. If the top of an $R^{\prime}$-tile touches $\left[u^{\prime}, u^{\prime \prime}\right]$, then the height of (at least) one of its vertical sides is again $M$, which implies the existence of another critical block which contains the new $R^{\prime}$-tile. Moreover, the new critical block is necessarily covered by the previous one.

The only remaining possibility is that $\left[u^{\prime}, u^{\prime \prime}\right]$ is the top side of an $m m^{\prime} \times n$ rectangle, tiled by $R$-tiles of $T_{\text {crit }}$ (since $M$ cannot be reached in a vertical side of an $R$-tile, the height of vertical sides of $R$-tiles of $T_{c r i}$ touching $\left[u^{\prime}, u^{\prime \prime}\right]$ is $M-2$, which gives the precise position of $R$-tiles). But, in this last possibility, the union of the block and the $m m^{\prime} \times n$ rectangle seen above form an $m m^{\prime} \times\left(n+n^{\prime}\right)$ rectangle on which a pushing down flip can be done, which is a contradiction (either this flip is height stable, or it creates a tiling covered by $\left.T_{\text {crit }}\right)$. This last case cannot hold. 
This study implies that there exists a block whose bottom side reaches $\delta F$ (since otherwise an infinite sequence of blocks would be generated), which yields that $M^{\prime}=$ $M-1$ and $M^{\prime}$ is reached on the lower boundary of $F$.

It remains to prove that $M^{\prime}$ is reached on the upper boundary of $F$. To do it, from $T_{\text {crit }}$, while it is possible, make a sequence of height stable pushing up flips. A new minimal tiling $T_{\text {subcrit }}$ is obtained by this way, and the maximal height reached by $T_{\text {subcrit }}$ is also $M$. The arguments used for $T_{\text {crit }}$ can be used in a symmetric way for $T_{\text {subcrit }}$ to prove that $M-1$ is reached on the upper boundary of $F$. This achieves the proof.

Of course, there exists a symmetric proposition when $M$ is even.

Corollary 1 We keep the notations above. Assume that $M^{\prime}$ is even. For each vertex $v$ $\delta F$ such that $h 1(v)=M^{\prime}$, at least one of the following alternatives holds:

* the vertex $v$ is on the boundary of an $R$-tile of $T_{\text {crit }}$ with two vertical sides of height $M^{\prime}-1$,

* the vertex $v$ is on the boundary of the horizontal side of a critical block of $T_{\text {crit }}$. Moreover, if $v$ is on the upper boundary of $F$, then there exists a stair $\left(B_{1}, B_{2}, \ldots, B_{p}\right)$ of critical blocks such that the block $B_{1}$ meets the lower boundary of $F$ and $v$ is on the top side of $B_{p}$.

Proof. Obvious, since any other situation contradicts the above proposition.

\subsection{Algorithm of tiling}

We present our algorithm below:

Initialization: Construct $f 1$ and $f 2$ on $\delta F$ (if a contradiction holds, then stop: there is no tiling)

Main loop: Consider the set $S_{M^{\prime}}$ of vertices such that $h 1(v)=M^{\prime}$. We assume that $M^{\prime}$ is even (the case when $M^{\prime}$ is odd is treated in a symmetric way).

If $S_{M^{\prime}}$ is included in the lower boundary of $F$, then place the $R$-tile $t$ in the neighborhood of on a vertex $v$ of $S_{M^{\prime}}$ in such a way that the maximal height reached in the boundary of $t$ is $M^{\prime}$ (at most one possibility with no contradiction for $f 1_{T}$ and $f 2_{T}$ ). Otherwise, take a vertex $v$ of $S_{M^{\prime}}$ in the upper boundary of $F$. If there exists a stair $\left(B_{1}, B_{2}, \ldots, B_{p}\right)$ of blocks such that the block $B_{1}$ meets the lower boundary of $F v$ is on the top side of $B_{p}$, and the stair creates no contradiction for $f 1_{T}$ and $f 2_{T}$, then place the tiles of the tiling of the stair of blocks. If such a stair does not exists, then place the $R$-tile $t$ in the neighborhood of the vertex $v$ in such a way that the maximal height reached in the boundary of $t$ is $M^{\prime}$ (at most one possibility with no contradiction for $f 1_{T}$ and $\left.f 2_{T}\right)$.

Remove the area tiled from $F$. Update $M^{\prime}$ and $S_{M^{\prime}}$. Repeat the main loop until the remaining figure is empty or a contradiction appears about tiling functions.

Corollary 2 Assume that $F$ can be tiled. The above algorithm gives a critical tiling. In particular, there exists a unique tiling $T_{\text {crit }}$. 
Proof. From Proposition 4, if $S_{M^{\prime}}$ is included in the lower boundary of $F$, then $M=M^{\prime}$, which guarantees the correctness of the placement of the tile in this case. On the other hand, Corollary 1 guarantees the correctness of the placement of the tiles when $S_{M^{\prime}}$ contains a vertex of the upper boundary of $F$.

From the above corollary, one can state the theorems below.

Theorem 1 (connectivity) Given any pair $\left(T, T^{\prime}\right)$ of tilings of a polygon $F$, one can pass from $T$ to $T^{\prime}$ by a sequence of flips.

Proof. Obvious.

Theorem 2 (quadratic algorithm) There exists a time quadratic tiling algorithm (in the area of the polygon $F)$ an algorithm which, given a polygon $F$, either produces a tiling or claims that there is no tiling in $O\left((A(F))^{2}\right)$ time units, where $A(F)$ denote the area of $F$.

Proof. we have seen in Corollary 2 that the algorithm given above is a tiling algorithm. We just have to study its complexity. Each passage through the loop permits to place at least one tile, thus there are at most $A(F)$ passages through the loop. Each passage through the loop costs at most $0(A(F))$ time units: the key-point is that the search of a the stair of blocks (in the discretized figure) is similar the depth first search in a tree which has at most $0(A(F))$ vertices. Thus the time complexity is at most quadratic in the area of $F$.

\section{Degenerate cases}

These cases when a tile has a side of unit length are degenerate, since at least one of the tiling groups is is degenerate, i. e. reduced to a cyclic group.

\subsection{A unique side of tile of unit length}

We now assume that $n=1$ and the other dimensions of tiles are not equal to 1 . With this hypothesis, $G_{2}$ is degenerate but, fortunately, $G_{1}$ is not degenerate. Thus we can apply Proposition 4, with the following modifications:

First modification: there is no possible stair of critical horizontal blocks, since, in this case, a horizontal block (which is an $m m^{\prime} \times n^{\prime}$ rectangle) admits two tilings which differ by a strong flip: if a critical block tiled by $R^{\prime}$-tiles would appear, then a decreasing strong flip could be done. Thus, when $M^{\prime}$ is even, we necessarily have $M=M^{\prime}$.

Second modification: if a vertical critical block $B^{\prime}$ covers another vertical critical block $B$, then $B^{\prime}$ is deduced from $B$ by a horizontal translation of length $m$. This yields that each stair of vertical critical blocks is formed with at most $m^{\prime}-1$ blocks (since we have to avoid subsequences of blocks forming an $m m^{\prime} \times n n^{\prime}$ rectangles).

How can the stairs of blocks crossing the polygon be now, detected ? since $G_{2}$ is degenerate, the method of the main case cannot be applied. Fortunately, in this case, stairs of blocks are quite simple. We use this simplicity as follows: 
An isthmus $I$ of $F$ is a $k m \times 1$ rectangle (where $k$ denotes an integer such that $0<k<m^{\prime}$ ) included in $F$, such that the left and right sides of this rectangle are respectively included in the left and right boundaries of $F$. For each tiling $T$ of $F$, there exists a pair $\left(\mu, \mu^{\prime}\right)$ of integers such that $I$ contains $\mu m$ cells covered by $R$-tiles and $\mu^{\prime} m^{\prime}$ cells covered by $R^{\prime}$-tiles. We have $\mu m+\mu^{\prime} m^{\prime}=k m$, which forces $\mu=k$ and $\mu^{\prime}=0$, since $k<m^{\prime}$. This proves that, in each tiling of $F$, each isthmus of $F$ is tiled with $R$-tiles. The above remarks yield to make the two modifications below on the tiling algorithm of the main case:

First modification: when $M^{\prime}$ is even, directly place an $R$-tile, since there cannot exist a vertical critical block.

Second modification: when $M^{\prime}$ is odd and $S_{M^{\prime}}$ contains a vertex of the left boundary of $F$ and a vertex of the right boundary of $F$, take a vertex $v$ of the right boundary. If $v$ is a corner of an isthmus of $F$, then tile the isthmus with $R$-tiles. Otherwise, place the unique $R^{\prime}$-tile whose a highest vertex is $v$.

With these modifications, each passage through the loop costs a bounded number of time units, which improves the complexity: in this case, we have a linear algorithm. We can also see that a pushing down (or pushing up) weak flips can be decomposed into a sequence of two strong flips. Thus the space of tilings is connected if we only use strong flips, pushing left and pushing up weak flips.

\subsection{Two opposite sides of unit length}

We now assume that $m^{\prime}=n=1, m^{\prime}>1$ and $n=1$. Thus $G_{1}$ remains not degenerate. In this case, there cannot exist any critical block, and we necessarily have $M=M^{\prime}$. Thus, in the algorithm, we do not have to search stairs of critical blocks, which improves the algorithm and gives a linear time complexity. We have found again the linear algorithm of [4]. We also find the connectivity result by strong flips, remarking that, in this case, each weak flip can be decomposed into a sequence of two strong flips.

\subsection{Two parallel sides of unit length}

We now assume that $n=n^{\prime}=1$. The problem (which is in fact one-dimensional) is reduced to a set of instances of the following problem (called the small problem): given an integer $k$, find a tiling of the $k \times 1$ rectangle. This small problem can be treated by the following algorithm: if $k$ is a multiple of $m$, then tile the whole rectangle with $R$-tiles. Otherwise, place a $R^{\prime}$ tile on the left part of the rectangle. Update the rectangle which remains to be tiled. Repeat these instructions until either the rectangle obtained is empty or an impossibility appears. Thus the small problem can be solved in $0(k)$ time units, thus the original problem can be solved in linear time in the area of the polygon.

The flip connectivity is easy: from any tiling of the rectangle, first make the necessary weak flips in order to place the $R^{\prime}$-tiles on the left part and the $R$-tiles on the right part. Afterwards, while there is at least $m R^{\prime}$-tiles, make a strong flip on the rectangle tiled with the $m$ rightmost $R^{\prime}$-tiles. The tiling obtained after the above flips is always the tiling given by the previous algorithm, which gives the connectivity. 


\subsection{A rectangle is reduced to a cell}

We now assume that $m=n=1$. In this case, we have a trivial algorithm of tiling (which only uses $R$-tiles) and a connectivity with strong flips.

\section{Extensions and open problems}

The arguments given in this paper seem to be extended to the case when dimensions are real numbers. It is more difficult to speak about algorithms and complexity in this framework, the goal is to find a method to construct a tiling, and a theorem of connectivity.

Another direction of research is a better knowledge of the structure of the space of tilings, i. e. the graph whose vertices are tilings of a polygon $F$ and two tilings are linked by an edge if and only if they only differ by a single flip. Some very strong structural results (lattice structures, effective computations of of geodesics between fixed tilings) have previously be found in the special case when each tile has a side of unit width [13] [14]. Can we extend these results to the main case ?

\section{References}

[1] D. Beauquier, M. Nivat, E. Remila, J. M. Robson, Tiling figures of the plane with two bars Computational Geometry: Theory and Applications 5, (1995) p. 1-25,

[2] J. K. Burton, C. L. Henley, A constrained Potts antiferromagnet model with an interface representation, J. Phys. A 30 (1997) p. 8385-8413.

[3] J. H. Conway, J. C. Lagarias, Tiling with Polyominoes and Combinatorial Group Theory, Journal of Combinatorial Theory A 53 (1990) p. 183-208.

[4] C. Kenyon, R. Kenyon, Tiling a polygon with rectangles, proceedings of the $33^{\text {rd }}$ IEEE conference on Foundations of Computer Science (FOCS) (1992) p. 610-619.

[5] R. Kenyon, A note on tiling with integer-sided rectangles, Journal of Combinatorial Theory A 74 (1996) p. 321-332.

[6] J. C. Lagarias, D. S. Romano, A Polyomino Tiling of Thurston and its Configurational Entropy, Journal of Combinatorial Theory A 63 (1993) p. 338-358.

[7] W. Magnus, A. Karrass, D. Solitar Combinatorial Group Theory. Presentation of groups in terms of generators and relations. $2^{\text {nd }}$ edition (1976), Dover publications.

[8] C. Moore, I. Rapaport, E. Rémila, Tiling groups for Wang tiles, proceedings of the $13^{\text {th }}$ annual ACM-SIAM Symposium On Discrete Algorithms (SODA) SIAM eds, (2002) p. 402-411.

[9] I. Pak, Ribbon Tile Invariants, Trans. Am. Math. Soc. 63 (2000) p. 5525-5561.

[10] I. Pak, Ribbon Tile Invariants, New Horizons, to appear in Theoretical Computer Science. 
[11] J. G. Propp, A Pedestrian Approach to a Method of Conway, or, A Tale of Two Cities, Mathematics Magazine 70 (1997), p. 327-340. 1997

[12] E. Rémila, Tiling groups : new applications in the triangular lattice, Discrete and Computational Geometry 20 (1998), p. 189-204.

[13] E. Rémila, An algebraic method to compute a shortest path of local flips between two tilings, proceedings of the $11^{\text {th }}$ annual ACM-SIAM Symposium On Discrete Algorithms (SODA) SIAM eds, Philadelphia, (2000) p. 646-653.

[14] E. Rémila, On the structure of some spaces of tilings, preprint, to appear in SIAM journal of Discrete Mathematics.

[15] S. Sheffield, Ribbon tilings and multidimensional height function, preprint arXiv:math.CO/0107095.

[16] W. P. Thurston, Conway's tiling groups, American Mathematical Monthly 97 (1990) p. 757-773. 